\title{
Axonogenesis in the Brain of Zebrafish Embryos
}

\author{
Ajay B. Chitnis ${ }^{1}$ and John Y. Kuwada ${ }^{1,2}$ \\ 'Neuroscience Program, ${ }^{2}$ Department of Biology and Institute of Gerontology, University of Michigan, Ann Arbor, \\ Michigan 48109
}

\begin{abstract}
We analyzed the pattern and development of the earliest tracts and followed pathfinding by the growth cones of an identified cluster of neurons in the brain of zebrafish embryos. Neurons were labeled with an antibody which labels many embryonic neurons, a lipophilic axonal tracer dye, and intracellular dye injections. The embryonic brain is extremely simple, and at $28 \mathrm{hr}$ of development, the forebrain and midbrain consist of 8 main axonal tracts which are arranged as a set of longitudinal tracts connected by commissures. Each tract is established by identified clusters of approximately 2-12 neurons found in discrete regions of the brain. Many identified clusters of neurons project axons in a defined direction appropriate for the cluster and have axons with stereotyped trajectories, suggesting that their growth cones follow cell-specific routes. This was confirmed with intracellular dye injections for neurons of the nucleus of the posterior commissure. The growth cones of these neurons arrive at a site in the anterior tegmentum where 4 tracts meet. At this site, they could, in principle, turn in a number of directions but always extend posteriorly into one of the tracts. The pattern of pathfinding by these growth cones suggests the testable hypothesis that the growth cones of identified clusters of neurons establish the simple set of early tracts by selecting cluster-specific pathways at such intersections in order to reach their targets in the brain.
\end{abstract}

Pathfinding by growth cones has been analyzed in a number of different regions within the nervous system in a variety of animals (reviewed in Cowan et al., 1984; Goodman et al., 1984; Palka, 1986; Dodd and Jessell, 1988; Patterson, 1988). From these studies a number of different mechanisms which guide growth cones and/or affect the final trajectories of axons have been identified. These include permissive interactions, specific interactions, and inhibitory interactions between growth cones and environmental cues; long distance cues; and the projection and elimination of exuberant axons. An understanding of why one mechanism is adopted over another may involve knowing the differences in conditions under which different mechanisms are operative. In this regard, the analysis of pathfinding by growth cones in the vertebrate brain may be instructive as well as chal-

\footnotetext{
Received May 30, 1989; revised Dec. 19, 1989; accepted Dec. 26, 1989.

We thank Gianni Piperno for the generous gift of the acetylated tubulin monoclonal antibody and R. Bernhardt, H. Okamoto, S. Wilson, R. Hume, and K. Tosney for critically reading the manuscript. Supported by grants from NIH (NS24848) and the University of Michigan Office of the Vice President for Research and a Basil O'Connor Award from the March of Dimes Birth Defects Foundation to J.Y.K

Correspondence should be addressed to John Y. Kuwada. Institute of Gerontology. University of Michigan, 300 N. Ingalls, Ann Arbor, MI 48109-2007.

Copyright $c 1990$ Society for Neuroscience $0270-6474 / 90 / 061892-14 \$ 02.00 / 0$
}

lenging since it consists of so many different neurons which develop in a highly complex spatial and temporal pattern. Thus, the growth cones of brain neurons must encounter a myriad of conditions as they navigate through the developing brain.

One strategy to investigate pathfinding in the vertebrate brain is to analyze the brains of relatively simple vertebrates. This approach was taken in the early 20 th century by investigators such as Coghill $(1926,1930)$ and Herrick $(1937,1938)$ and recently by Roberts et al. (1987) and Easter and Taylor (1989) to study the development of the amphibian brain. These investigators found that the early vertebrate brain is surprisingly simple, being composed of a relatively small number of axonal tracts found in a stereotyped pattern not unlike the simple, orthogonal pattern of tracts found in nematode larvae (Albertson and Thomson, 1976; White et al., 1976) and insect embryos (Thomas et al., 1984). However, in these studies it was difficult to characterize the neurons which established the early tracts, and pathfinding by growth concs within these carly tracts was not determined. Consequently, there is little characterization of pathfinding by growth cones which takes into account the pathways which are potentially available to them in the vertebrate brain. Such a description of pathfinding by a particular set of growth cones would indicate the precision of axonogenesis by identifying which pathways are bypassed and which are followed as they navigate through the vertebrate brain to their termination sites.

One characteristic shared by a variety of neurons in the mammalian brain is the projection of exuberant axons which are later eliminated to establish the mature trajectories of these neurons (Cowan et al., 1984). For example, pyramidal cells in the rat occipital cortex project collateral axons to the superior colliculus, pontine nuclei, and spinal cord during embryogenesis. The corticospinal projection is lost during the first 2 weeks following birth while the other collaterals are retained (Stanfield et al., 1982; Stanfield and O'Leary, 1985). This contrasts with axonogenesis by neurons in the CNS of insect embryos (Goodman et al., 1984) and spinal neurons in chick and fish embryos (Eisen et al., 1986, 1989; Kuwada, 1986; Landmesser, 1988; J. Y. Kuwada, R. R. Bernhardt, and N. Nguyen, unpublished observations). These neurons project growth cones which follow stereotyped pathways to reach their targets and during embryogenesis do not extend exuberant axons to inappropriate sites.

We have investigated the pattern of early axonal tracts and pathfinding by identified clusters of neurons in the brain of zebrafish embryos in order to address 2 issues. (1) Is there a simple, orthogonal array of axonal tracts in the brains of early zebrafish embryos as exists in the early CNS of nematodes, insects, and amphibians? What is the identity of the neurons which establish the earliest brain tracts? (2) How do the growth 

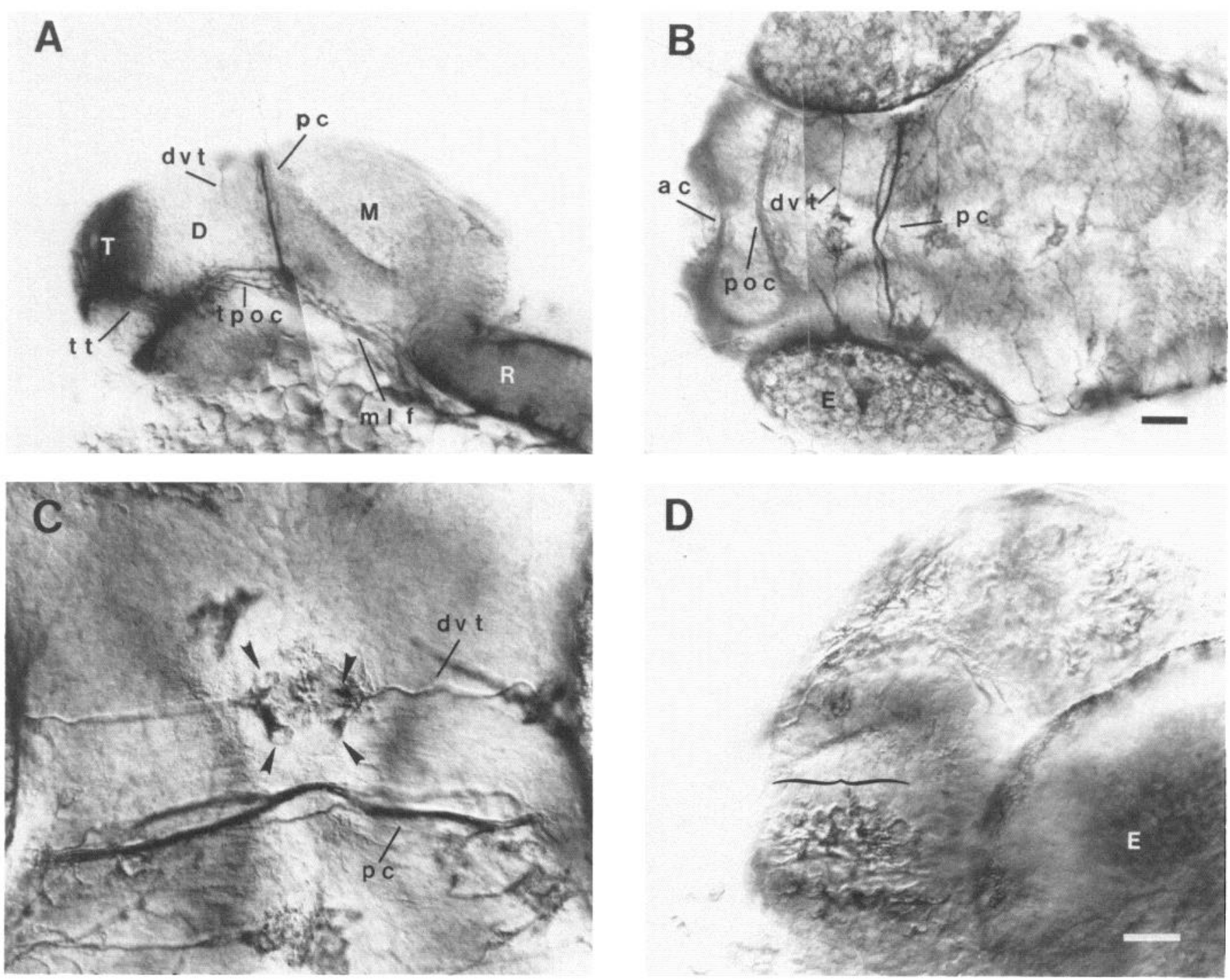

Figure 1. Simple pattern of embryonic brain tracts from $28 \mathrm{hr}$ whole-mounted zebrafish labeled with a monoclonal antibody to acetylated tubulin. $A$, Side view of the brain. Eyes were dissected away. Material ventral to the brain are yolk granules. Unless otherwise stated, in all figures anterior is left and dorsal is $u p ; T, D, M$, and $R$ are telencephalon, diencephalon, mesencephalon, and rhombencephalon, respectively; $t t$, telencephalic tract; $d v t$, diencephalic dorsoventral tract; $p c$, posterior commissure; $t p o c$, tract of the postoptic commissure; mlf, medial longitudinal fasciculus. $B$, Dorsal view. $A c$, anterior commissure; $p o c$, postoptic commissure. Scale bar (in $B$ ) for $A$ and $B, 40 \mu$ m. $C$, Higher-power view of the dorsal portion of the brain. Arrowheads point to neurons which project dvt axons. D, Rostral end of the brain. Neurons which project POC and TPOC axons are bracketed. $E$, eye. Scale bar (in $D$ ) for $C$ and $D, 20 \mu \mathrm{m}$.

cones of these early neurons extend to their termination sites? Do they bypass some tracts but follow others? Do they, like some mammalian neurons, project exuberant axons which are later eliminated? When faced with a number of different routes at pathway intersections, do growth cones select cell-specific pathways? Here we describe the organization and development of the early brain tracts and the neurons which project axons into these tracts.

\section{Materials and Methods}

Animals. Zebrafish embryos were collected from a laboratory breeding colony and maintained according to the procedures described in Myers et al. (1986).

Antibody labeling. Embryos were labeled with a monoclonal antibody against acetylated tubulin (Piperno and Fuller, 1985) as whole-mounts by following the procedures for whole-mount labeling described in Patel et al. (1989). Lucifer yellow (LY)-injected neurons were in some cases marked with HRP reaction product by application of a rabbit antiserum to LY dye (Taghert et al., 1982). Following intracellular injections, embryos were fixed in $2-4 \%$ paraformaldehyde and $1 \%$ dimethyl sulfoxide (DMSO) in $75 \mathrm{~mm}$ phosphate buffer ( $\mathrm{pH} \mathrm{7.4)}$ for $30 \mathrm{~min}$, washed in buffer; immersed in 0.5-1\% collagenase (Sigma, Type 1A) in phosphate buffer for 3-5 min; washed; and incubated in 1:500 dilution of LY antiserum with $0.5-1 \%$ Triton X-100, 0.5-1\% BSA, and $0.5-1 \%$ normal goat serum in phosphate buffer overnight at $5^{\circ} \mathrm{C}$. Embryos were then washed; incubated in 1:250 dilution of HRP-conjugated goat antirabbit with $0.5-1 \%$ Triton $\mathrm{X}-100,0.5-1 \% \mathrm{BSA}$, and $0.5-1 \%$ normal goat serum overnight at $5^{\circ} \mathrm{C}$ or for $3-5 \mathrm{hr}$ at $33^{\circ} \mathrm{C}$; washed; incubated in diaminobenzedine $(0.5 \mathrm{mg} / \mathrm{ml})$ for $20 \mathrm{~min}$; and reacted by adding hydrogen peroxide. In some cases, antibody-labeled embryos were refixed, dehydrated, embedded in plastic, and sectioned ( $1 \mu \mathrm{m}$ semi-thins). Electron microscopy. Embryos were processed for electron microscopy (EM) by fixing in 3\% glutaraldehyde, $2 \%$ paraformaldehyde, $1 \%$ acrolein, and 1\% DMSO in $75 \mathrm{~mm}$ phosphate buffer for $1 \mathrm{hr}$; washed; postfixed in $2 \%$ osmium tetroxide in phosphate buffer for $2 \mathrm{hr}$; washed in 50 mм sodium maleate buffer ( $\mathrm{pH} 5.9$ ); stained with $2 \%$ uranyl acetate in maleate buffer for $1-2 \mathrm{hr}$; washed in maleate buffer; dehydrated in 

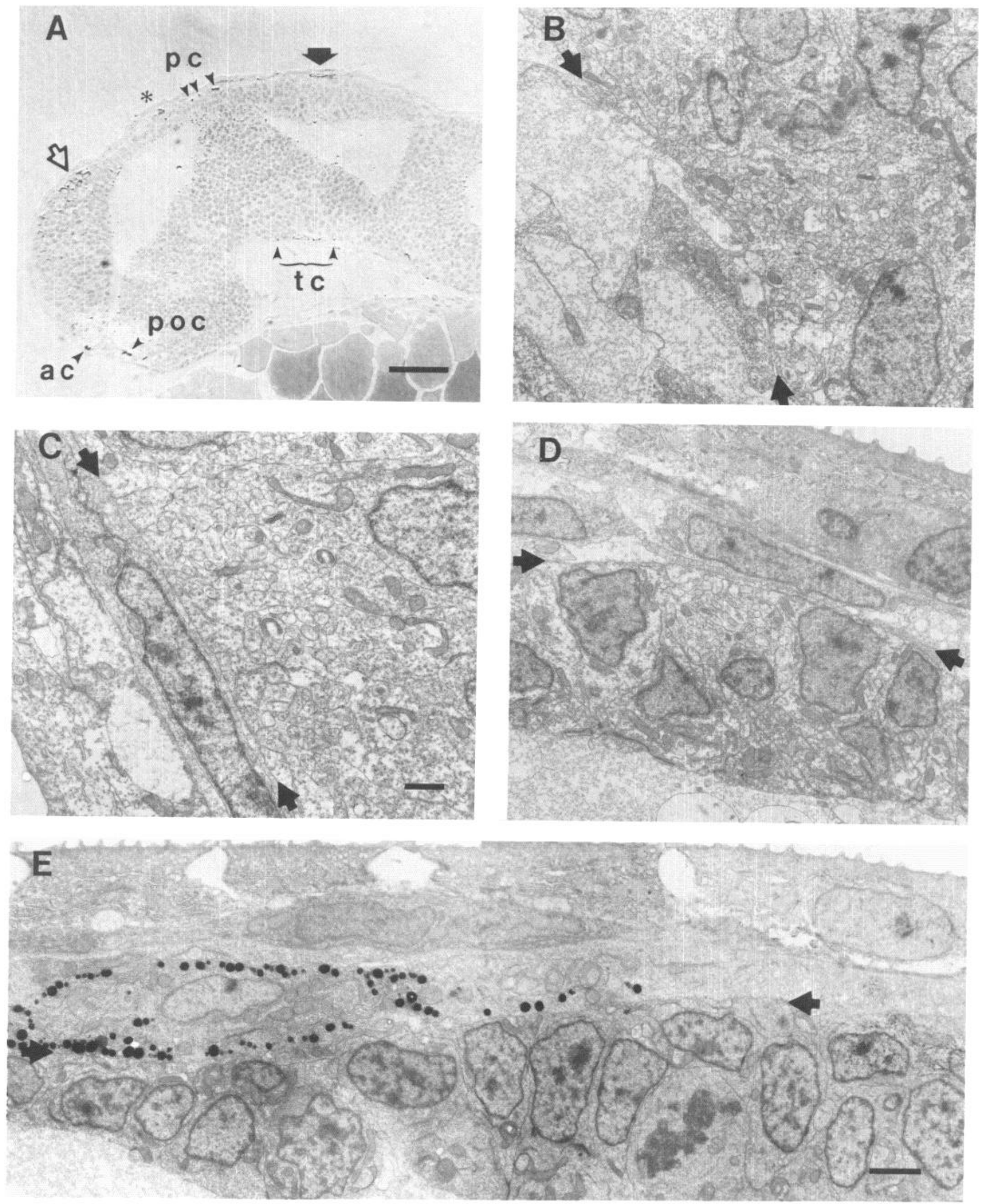

Figure 2. Acetylated tubulin antibody labels brain tracts in a $28 \mathrm{hr}$ embryo. $A$, Sagittal section near the midline of the brain. Occasional labeling near the ventricles are cilia. Melanophores in the skin such as the one under the closed arrow show up as dark patches. Open arrow, telencephalic neurons; asterisk, dvt neurons; closed arrow, region of the tectum which is shown in $E$; $t$, tegmental commissure; all other abbreviations as in Figure 1. Scale bar, $40 \mu \mathrm{m}$. B, Electron micrograph of the postoptic commissure. In this and the following panels, basal lamina at the edge of the brain can be seen in between the arrows. $C$, Anterior commissure. Scale bar, $1 \mu \mathrm{m}$. $D$, Posterior commissure. $E$, Dorsal region of the tectum denoted by closed arrow in $A$. Note the melanophore which is often found in this region. Scale bar (in $E$ ) for $B, D$, and $E, 2 \mu \mathrm{m}$. 


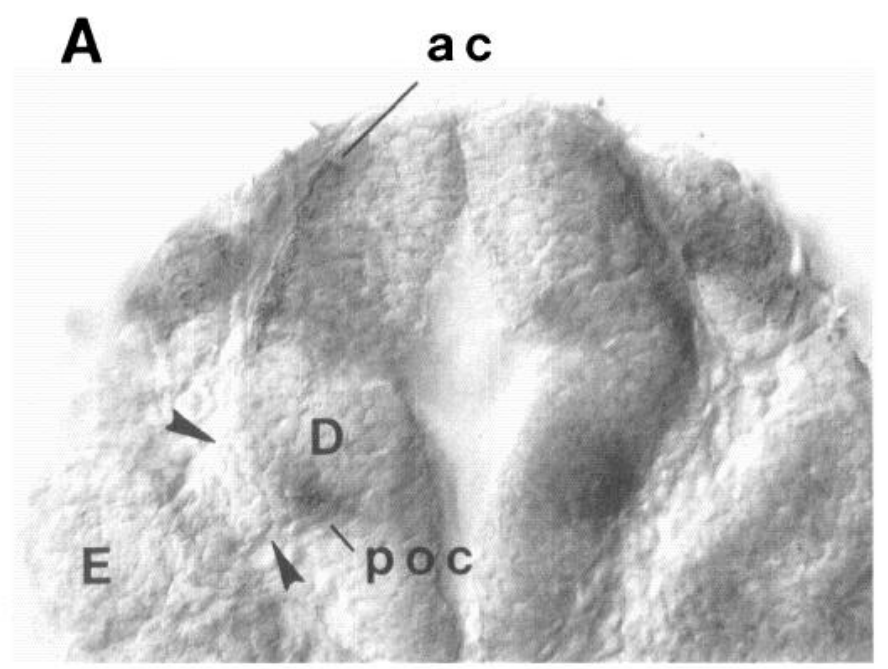

B

Figure 3. Identification of the anterior and postoptic commissures, the olfactory nerve, and the nascent olfactory bulb. Micrographs of ventral/ dorsal view of the rostral brain labeled with the acetylated tubulin antibody from a $32 \mathrm{hr}$ embryo. $A$, The anterior and postoptic commissures are anterior and posterior, respectively, to the optic stalk, which on the left side is denoted by the arrowheads and runs from the eye ( $E$ ) to the diencephalon $(D)$. Anterior, $u p$. $B$, More dorsal view showing the olfactory nerve $(O N)$ running from the nasal placode to the nascent olfactory bulb in the telencephalon. Scale bar (in $B$ ) for $A$ and $B, 40 \mu \mathrm{m}$.

an ethanol and propylene oxide series; embedded in plastic; and thinsectioned.

DiI labeling. Embryos were dissected to remove their yolk sacs, pinned in a Sylgard (Dow Corning)-lined embryo holder, fixed for $6 \mathrm{~min}$ to 8 $\mathrm{hr}$ in $2-4 \%$ paraformaldehyde and $1 \%$ DMSO in phosphate buffer, washed, and placed on a fixed-stage Zeiss compound microscope fitted with differential interference contrast (DIC) optics and epifluorescence. Neurons were labeled from their axons by pressure injection $(80 \mathrm{psi}$, $100 \mathrm{~ms}$ pulse) of a small amount of diI, $1,1^{\prime}$-dioctadecyl-3,3,3',3'-tetramethylindocarbocyanine perchlorate (Molecular Probes, $0.25 \%$ in $N, N$-dimethylformamide) (Honig and Hume, 1986), from a microelectrode under visual control in a discrete area of the brain. DiI was allowed to spread for $1-12 \mathrm{hr}$ and the labeled neurons visualized, photographed, or drawn with the aid of a camera lucida. In some cases, diI-labeled neurons were marked with a brown reaction product by following the photooxidation procedure with diaminobenzedine and epifluorescent illumination (Maranto, 1982). The number of neurons back-labeled with diI was variable as with many other back-labeling techniques.

LY injections. Embryos were prepared as described above for dil labeling. Neuronal somata were visualized with DIC optics, impaled with microelectrodes filled with $10 \% \mathrm{LY}$ in distilled water, and filled with dye by injecting hyperpolarizing currents $(0.1-0.5 \mathrm{nA}$ for $10-120$ sec). Labeled neurons were viewed with epifluorescence and photographed and /or drawn with the aid of a camera lucida. In come cases, LY-labeled neurons were secondarily marked via the photooxidation procedure or with the LY antiserum followed by a HRP-labeled secondary antibody.

\section{Results}

Antibody to acetylated tubulin labels the earliest tracts in the zebrafish brain

A method for consistent labeling of neurons in embryonic nervous systems can be extremely useful for studies of axonogenesis. For example, an antibody against HRP, which appears to label all insect embryonic neurons, was used to identify many of the early peripheral neurons of insect embryos and to delineate how their growth cones navigate through the embryo to reach their targets (Jan and Jan, 1982; Caudy and Bentley, 1986a, b; Blair et al., 1987). Likewise, a monoclonal antibody directed against acetylated tubulin (Piperno and Fuller, 1985) labels many of the embryonic axons in the zebrafish brain (see below; Fig. 1). This antibody labels cilia, ependymal processes, axons, and the cell bodies of neurons which have begun axonogenesis. It is likely that embryonic axons are labeled to the base of growth cones since broad, unlabeled processes can be seen at the distal end of labeled axons with DIC optics (data not shown). Labeled axons are readily distinguishable from cilia or ependymal processes because the earliest axons are located near the superficial surface of the brain (Fig. 2), and neuronal cell bodies are relatively large and also found superficially in the early brain (Fig. 1). Cilia are limited to the lumenal side of the brain and long ependymal processes run radially from the lumenal surface to the outer margin.

To determine whether the antibody labels many if not all the axonal tracts present in the early brain, we first compared the pattern of labeling in semi-thin sagittal sections of labeled zebrafish brains $(n=4)$ with the pattern of axons in corresponding EM sections from unlabeled brains ( $n=3$; Fig. 2). Inspection of the entire brain with the electron microscope revealed discrete bundles of profiles located in stereotyped positions in the early brain. These profiles contained microtubules and were assumed to be bundles of axons. All the axon bundles (anterior, postoptic, posterior, and tegmental commissures) in the EM sections corresponded to those recognized by the antibody. In other areas, such as the dorsal region of the tectum, no antibody labeling was seen at $28 \mathrm{hr}$. In EM sections of the same area no axonal profiles were evident (Fig. $2 E$ ), although at much later stages axons will occupy this region (data not shown). Second, we compared the brain tracts labeled following large applications of the lipophilic dye, diI (Honig and Hume, 1986), into various regions of the brain with the pattern of tracts labeled by the antibody in $28 \mathrm{hr}$ embryos. These diI applications revealed the same set of tracts as the antibody (see below). In no case was there a tract labeled by diI which was not labeled by the antibody. These data suggest that the pattern of tracts revealed by the antibody represents all the axonal bundles present in the embryonic brain. Our data do not exclude the possibility that some axons in a tract, or scattered single axons not in tracts, may not be labeled by the antibody. However, the antibody labeled all the neuronal cell types of the embryonic zebrafish 


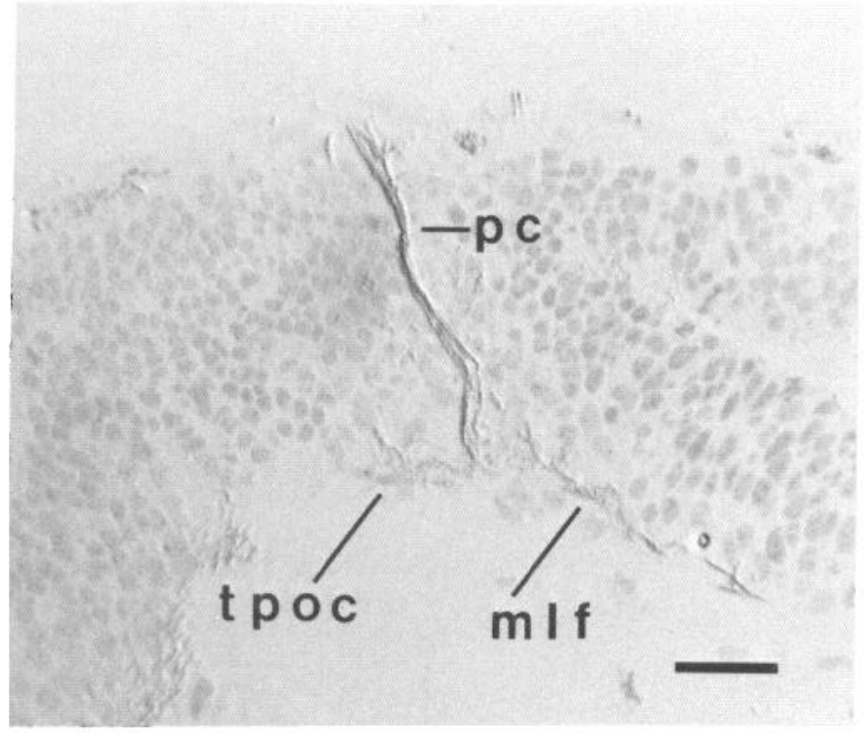

Figure 4. Intersection of the posterior commissure, TPOC, and MLF in the anterior tegmentum. Parasagittal section of $28 \mathrm{hr}$ brain labeled with acetylated tubulin antibody. Scale bar, $40 \mu \mathrm{m}$.

cord which had been identified independently by intracellular dye injections and by back-labeling with axonal tracer dyes $(\mathrm{R}$. R. Bernhardt, A. B. Chitnis, L. Lindamer, and J. Y. Kuwada, unpublished observations).

\section{Organization of the early brain tracts}

Eight main axonal tracts were labeled with the acetylated tubulin antibody in the forebrain and midbrain of $28 \mathrm{hr}$ embryos $(n=$ 27; Figs. 1, 2). We identified these tracts by comparisons with the tracts in adult fishes (Johnston, 1911; Ariens Kappers et al., 1936) and with the early brain tracts in amphibia (Coghill, 1926, 1930; Herrick, 1937, 1938). When the embryonic tracts could not be identified unambiguously by these comparisons, they were named by their location. The 8 tracts are the anterior commissure, telencephalic tract, postoptic commissure, tract of the postoptic commissure (TPOC), medial longitudinal fasciculus (MLF), posterior commissure, ventral tegmental commissure, and the dorsoventral diencephalic tract.

The anterior commissure of the zebrafish embryo was identified by homology to the anterior commissure in the amphibian embryo (Herrick, 1938). It is located at the rostral end of the brain anterior to the optic stalk in the telencephalon (Fig. 3). At this time, the nascent olfactory bulbs have not evaginated from the cerebrum but are identifiable as the termination sites of the olfactory nerves (Figs. 1, 3). The telencephalic tract consists of axons which run from the telencephalon to the ventral diencephalon, where it connects with the TPOC. We identified the postoptic commissure by homology with the amphibian embryo (Herrick, 1938). The postoptic commissure is located just posterior to the optic stalk (Fig. $3 A$ ) and runs between the 2 sides of the anterior diencephalon (Fig. $1 B$ ). The postoptic commissure is contiguous with the TPOC, which runs caudally through the ventral diencephalon, where it breaks up into several parallel axon bundles (Fig. $1 A$ ). These bundles run into the anterior tegmentum with the more ventral bundles contiguous with the MLF and the more dorsal ones running through the dorsal tegmentum and eventually merging into the dorsal longitudinal fasciculus (DLF) (see next section). The MLF is a
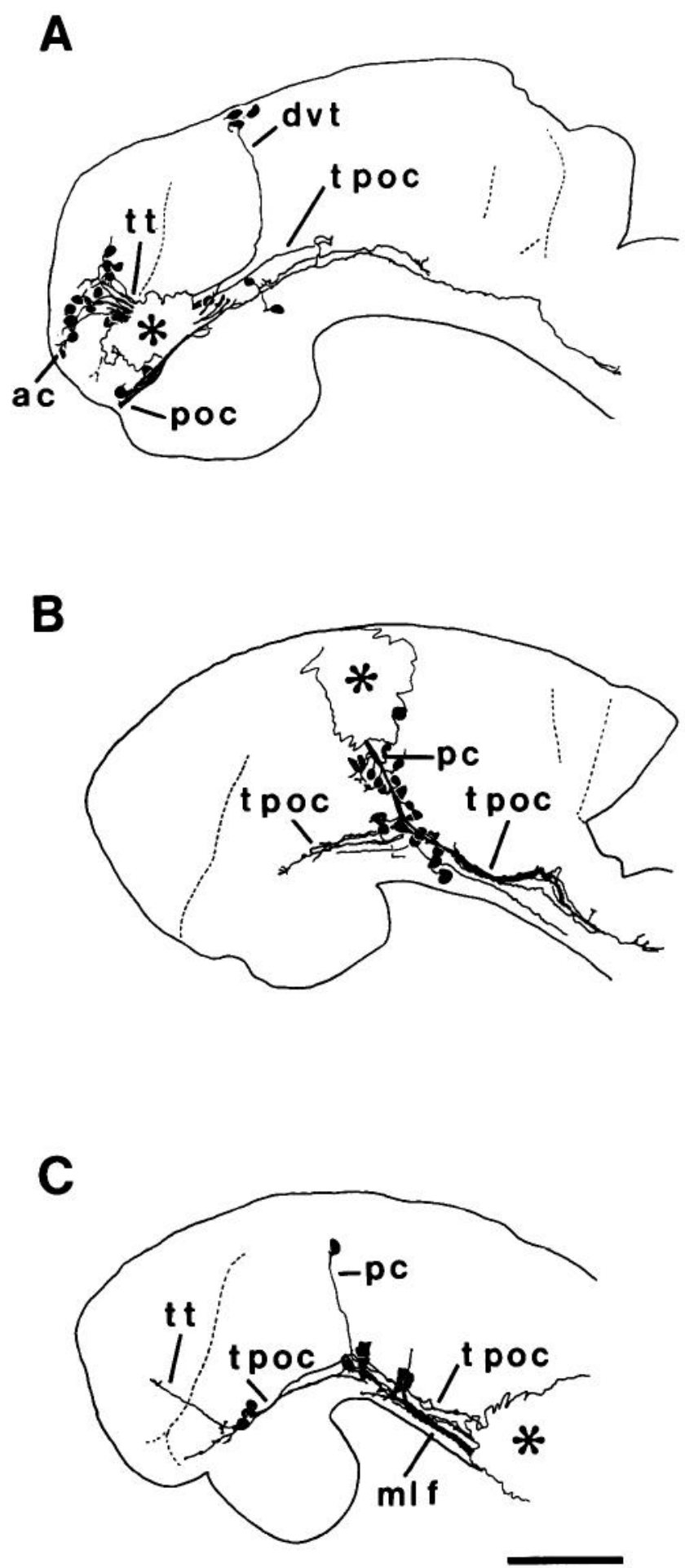

Figure 5. Early brain tracts labeled by large applications of diI in 28 hr embryos. In each case, camera lucida drawings were made following diI application, photooxidation, and removal of the eyes. $A$, DiI applied to the ventral diencephalon (asterisk) labeled axons in the anterior and postoptic commissures ( $a c$ and $p o c$ ), telencephalic tract $(t t)$, dorsoventral diencephalic tract ( $d v t)$, and the TPOC. $B$, DiI applied to the dorsal midline anterior to the tectum (asterisk) labeled axons in the posterior commissure $(p c)$ and TPOC. $C$, Dil applied to the posterior tegmentum/ anterior hindbrain (asterisk) labeled axons in the MLF, TPOC, PC, and TT. Scale (in $C$ ) for all panels, $100 \mu \mathrm{m}$. 
A

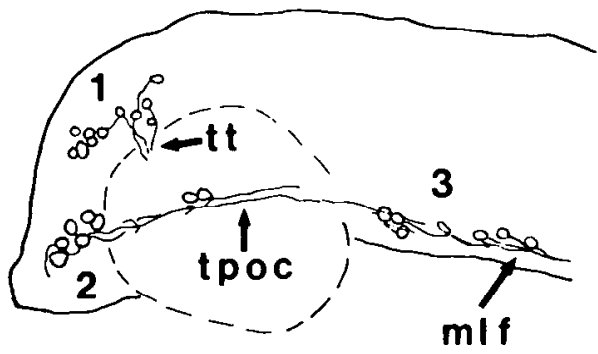

17

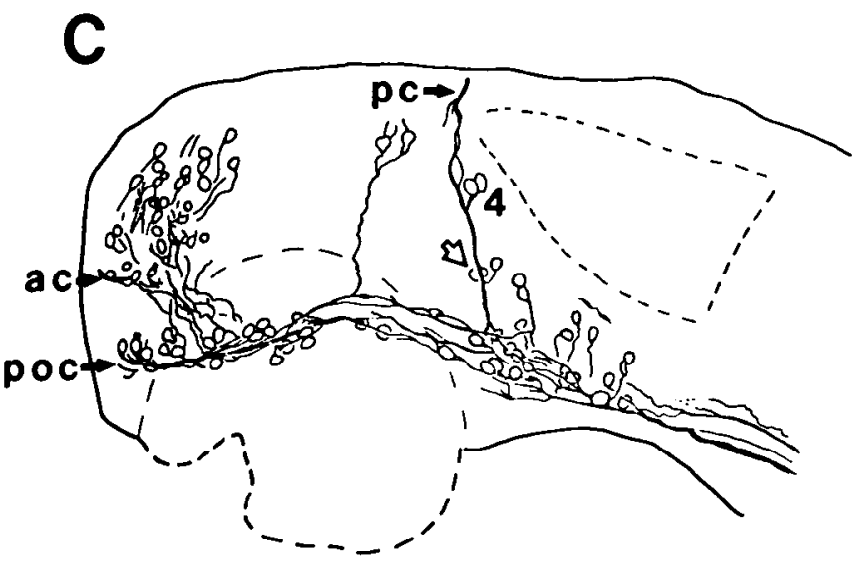

22
B

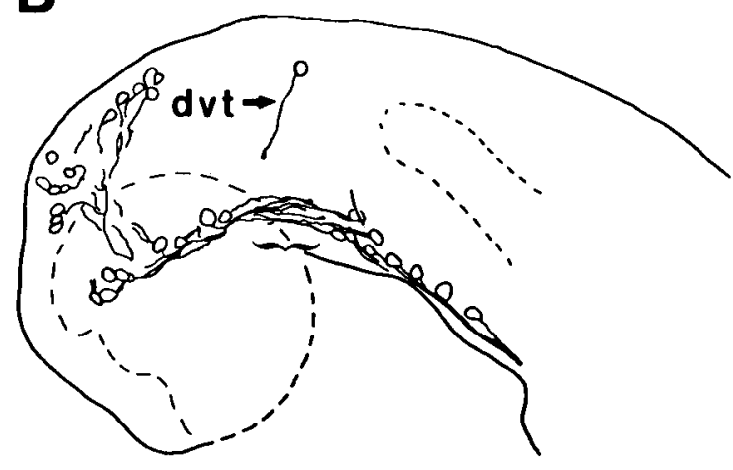

20

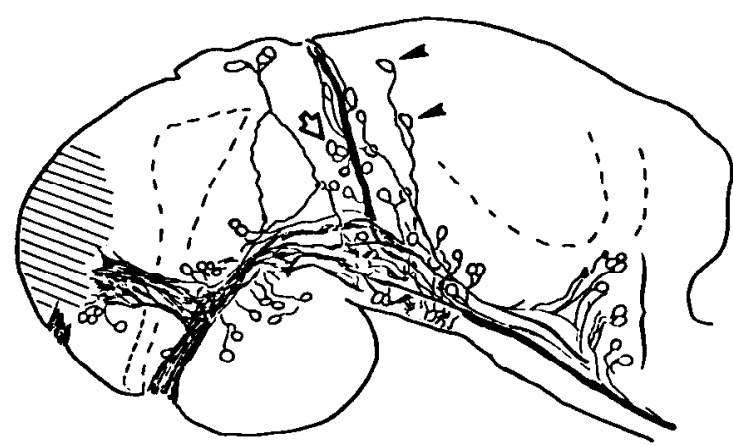

28

Figure 6. Sequence of development of the early brain tracts. Camera lucida drawings of brain axons in embryonic brains labeled with acetylated tubulin antibody. $A, 17 \mathrm{hr}$. Numerals 1,2 , and 3 denote the telencephalic neurons, postoptic neurons, and neurons of the nuc MLF, respectively. Eye is denoted by dashes. $B, 20 \mathrm{hr}$. The mesencephalic ventricle is denoted by shorter dashes; bracket denotes parallel bundles of the TPOC. $C$, $22 \mathrm{hr}$. The number 4 denotes the nuc PC; open arrow denotes posterior commissure neurons of the lateral cluster in the anterior tegmentum; bracket denotes tegmental commissure neurons. $D, 28$ hr. Eyes were dissected after labeling. Telencephalic neurons have not been drawn in the hatched region; arrows, early tectal neurons; bracket, cerebellar neurons. Scale (in $D$ ) for all panels, $100 \mu \mathrm{m}$.

conspicuous tract which runs along the medial floor of the brain from the anterior tegmentum through the hindbrain and eventually into the spinal cord (Kimmel et al., 1982).

The dorsoventral diencephalic tract originates from a small nucleus of neurons found near the dorsal midline of the diencephalon (Fig. 1C). At present, it is unclear whether these neurons are located in the lateral wall of the epiphysis or are in the diencephalon lateral to the epiphysis. This tract runs to the TPOC in the ventral diencephalon. The posterior commissure crosses the dorsal midline between the epiphysis and the anterior border of the tectum and runs ventrally to the floor of the anterior tegmentum, where it runs into the TPOC and MLF. The ventral tegmental commissure is found in the floor of the anterior tegmentum. In this portion of the anterior tegmentum, 4 different tracts (TPOC, posterior commissure, MLF, and ventral commissure) converge. Semi-thin parasagittal sections of $28 \mathrm{hr}$ brains labeled with the antibody clearly show the convergence of the TPOC, posterior commissure, and the MLF in the anterior tegmentum (Fig. 4).
Essentially the same pattern of tracts labeled by the acetylated tubulin antibody was also labeled by large applications of diI to different regions of the brain in $28 \mathrm{hr}$ embryos. Application of diI to the ventral diencephalon $(n=20)$ labeled axons in the TPOC, postoptic commissure, telencephalic tract, anterior commissure, and the diencephalic dorsoventral tract (Fig. 5A). Applications to the dorsal midline anterior to the tectum $(n=18)$ labeled axons in the posterior commissure and TPOC (Fig. $5 B$ ). Finally, diI applied to the posterior tegmentum/anterior hindbrain $(n=24)$ labeled axons in the TPOC, MLF, posterior commissure, and telencephalic tract (Fig. 5C).

\section{Sequence of development of the early brain tracts}

The development of the early tracts was followed by application of the acetylated tubulin antibody. Neurons undergoing axonogenesis can be readily traced from axons back to the cell bodies in the early brain since there are very few neurons projecting axons in the early stages (see below). The first axons are evident in the brain at $17 \mathrm{hr}$ of development $(n=23$; Fig. $6 A)$. These 

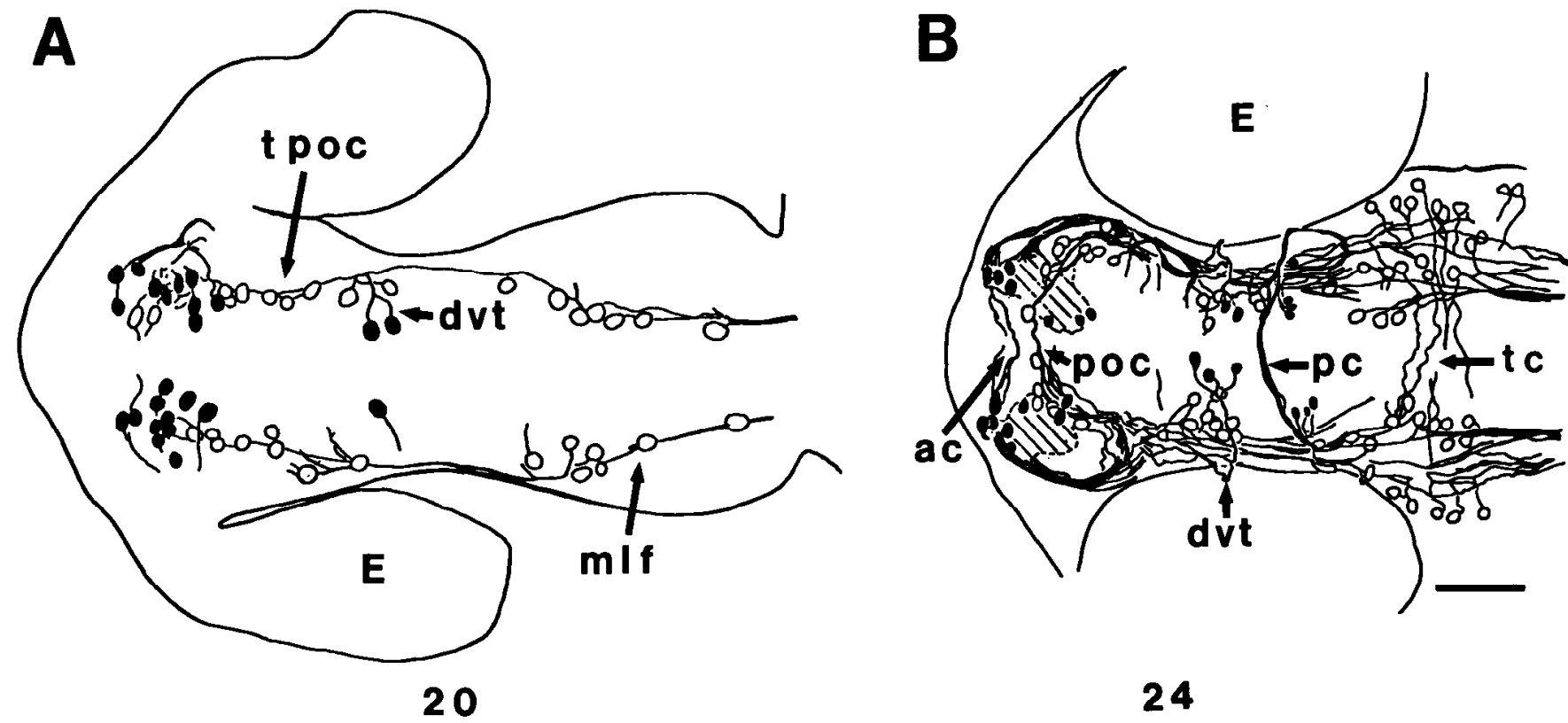

Figure 7. Sequence of development of the early brain commissures. Camera lucida drawings of embryonic axons labeled with acetylated tubulin antibody seen from a dorsal perspective. $A, 20 \mathrm{hr}$. Dorsal somata are filled in black; ventral somata are outlined; anterior, left. B, $24 \mathrm{hr}$. Hatched region contained telencephalic neurons which wcrc not drawn; brackets denote somata at tegmental commissure cells. Scale (in $B$ ) for $A$ and $B$, $50 \mu \mathrm{m}$.

axons pioneer the TPOC, MLF, and the telencephalic tract. At the same time, neurons in the nasal placode have projected axons to the olfactory region of the telencephalon to establish the olfactory nerve (data not shown). At $20 \mathrm{hr}(n=30)$ the dorsoventral diencephalic tract develops (Figs. $6 B, 7 A$ ). At 22$24 \mathrm{hr}(n=53)$ the anterior, postoptic, posterior, and ventral tegmental commissures have formed (Figs. 6C, 7B). By $28 \mathrm{hr}$ $(n=27)$, tectal and ccrcbcllar ncurons have projected axons (Fig. 6D). As the brain develops the rostral-most end curves ventrally and backward so that the optic stalk and the adjacent anterior and postoptic commissures are found in a more posterior position than at earlier stages (Fig. 3). The following description of the development of specific brain tracts was based on the antibody preparations referred to above.

\section{Development of the TPOC and MLF}

The TPOC is established by a group of approximately 8 neurons which are found in the anterior diencephalon ventral to the telencephalon at $17 \mathrm{hr}$ of development (Fig. 6 A). These postoptic neurons project axons which extend in a caudal direction along the outer surface of the ventral diencephalon towards the nucleus of the MLF (nuc MLF) in the anterior tegmentum. At the same time, approximately 6 neurons in the nuc MLF project axons in a caudal direction to pioneer the anterior-most section of the MLF. By $20 \mathrm{hr}$ of development, some of the TPOC axons have reached the anterior tegmentum (Figs. $6 B, 7 A$ ). In the anterior diencephalon, the TPOC is a single tract but caudally the axons of the TPOC diverge and tend to grow in distinct parallel bundles. In $23 \mathrm{hr}$ embryos, the ventral bundles can be seen to project into the MLF and the more dorsal bundles extend in the dorsolateral tegmentum (Fig. 8A). That TPOC ncurons project axons into the longitudinal tracts in the tegmentum was confirmed by intracellular I.Y injections ( $n=3$ cells; Fig. $8 B$ ) and diI backfills from the posterior tegmentum/anterior hind- brain (Fig. 5C) in 26-28 hr embryos. At $28 \mathrm{hr}$, the anterior portion of the TPOC is a thick bundle of axons. By this time several neurons in the anterior tectum have axons which run ventrally to the tegmentum and turn posteriorly into the TPOC, and cerebellar neurons located along the anterior border of the cerebellum have axons which run ventrally to join the longitudinal tracts in the hindbrain (Fig. 6D). Sometimes tectal axons can be seen to join the dorsal TPOC bundles as early as $23 \mathrm{hr}$ (Fig. 8A). In the hindbrain, the dorsal TPOC bundles merge into the DLF (data not shown).

\section{Development of the telencephalic tract}

Approximately 8-10 neurons located in the telencephalon project axons which establish the telencephalic tract at $17 \mathrm{hr}$ (Fig. $6 \mathrm{~A}$ ). The telencephalic axons extend along the outer surface of the telencephalon towards the ventral diencephalon to join the axons of the TPOC by $20 \mathrm{hr}$ (Fig. 6B). As development progresses, additional axons are added to the telencephalic tract so that by $28 \mathrm{hr}$ it is a very thick tract (Fig. 6, C, D). The telencephalic tract appears to be a major pathway connecting the telencephalon and the diencephalon and, therefore, may correspond to the lateral and medial forebrain bundles (Kuhlenbeck, 1977).

\section{Development of the dorsoventral diencephalic tract}

The dorsoventral diencephalic tract is pioneered by 1-2 neurons in the dorsal diencephalon by $20 \mathrm{hr}$ and as early as $18 \mathrm{hr}$ (Fig. $6 B$ ). These neurons project axons toward the TPOC in the ventral diencephalon. By $22-24 \mathrm{hr}$ the dorsoventral diencephalic axons have reached the TPOC posterior to the telencephalic tract (Fig. 6C). These axons traverse approximately $100 \mu \mathrm{m}$ along a pathway which is presumably devoid of any preexisting axons from the dorsal diencephalon to the TPOC. Unlike the other early tracts, the dorsoventral diencephalic tract remains 


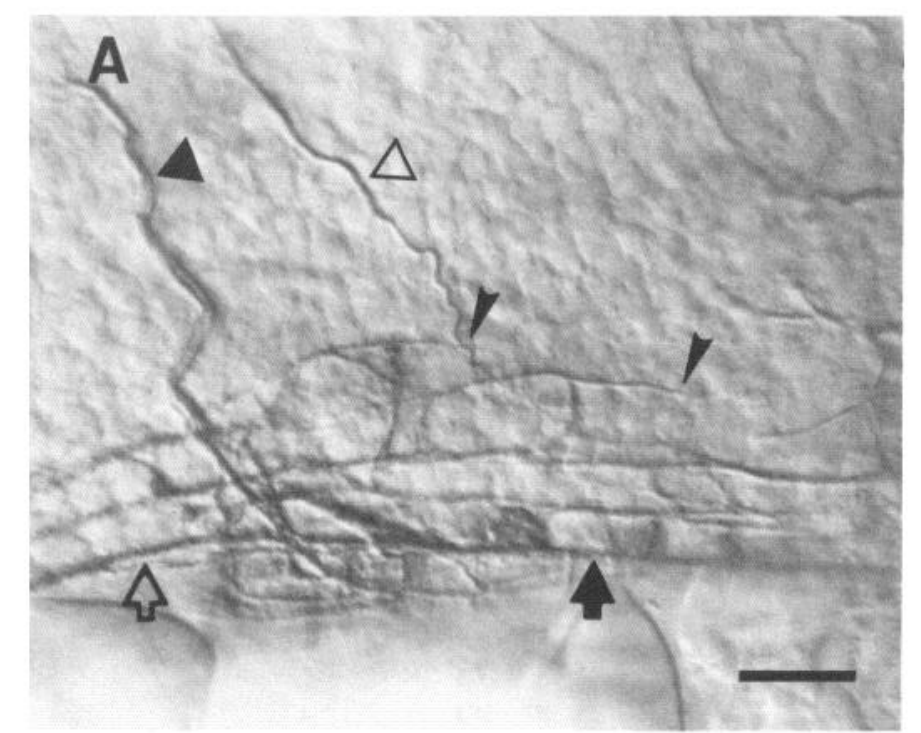

\section{B}

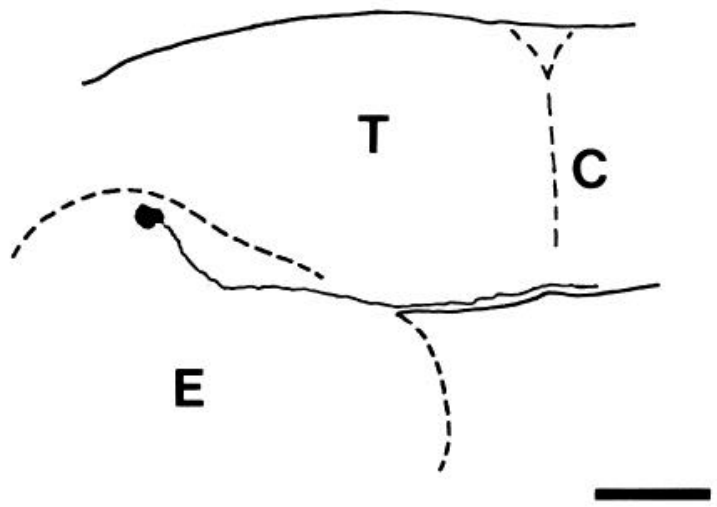

Figure 8. Parallel bundles of the TPOC and the MLF in the posterior diencephalon and anterior tegmentum. $A$, Micrograph of ventral TPOC bundle (open arrow) running into the MLF (closed arrow) and dorsal TPOC bundles (arrowheads) extending into the dorsolateral tegmentum of 23 $\mathrm{hr}$ embryo labeled with acetylated tubulin antibody. Closed triangle, posterior commissure; open triangle, early tectal axon. Scale bar, $20 \mu \mathrm{m}$. $B$, Drawing of LY-filled TPOC neuron in a $28 \mathrm{hr}$ embryo. $E$, eye; $T$, tectum: $C$, cerebellum. Scale bar, $40 \mu \mathrm{m}$.

a thin bundle of axons up to $28 \mathrm{hr}$ (Figs. $1 \mathrm{C}, 6 \mathrm{D}$ ). This tract is distinct from the habenular commissure. The habenular commissure develops later and is found just rostral to the epiphysis and the dorsoventral diencephalic tract in the $48 \mathrm{hr}$ embryo (data not shown). On rare occasions, the axons in this tract separate from each other before reaching the TPOC (Fig. 6D). In such cases, all the axons eventually reach the TPOC, with some axons having trajectories which suggest they had made course corrections to reach the TPOC.

\section{Development of the commissures}

The posterior, anterior, postoptic, and ventral tegmental commissures develop between 20 and $22-24 \mathrm{hr}$ (Figs. $6 C, 7 B$ ). The posterior commissure contains the axons of neurons in the anterior tegmentum (see next section) and the nucleus of the posterior commissure (nuc PC). The nuc PC is found in a dorsolateral position between the epiphysis and the anterior border of the nascent tectum and at these early stages contains approximately 2-3 neurons. These neurons have axons which course ventrally toward the anterior tegmentum.

The anterior and postoptic commissures form and connect the anterior regions of the telencephalon and the TPOCs on the 2 sides of the brain, respectively. Although the origin of the postoptic commissural axons is unclear, it is likely that some of the neurons in the anterior portion of the ventral diencephalon with TPOC axons project commissural axons as well since some of them are bipolar (data not shown). The ventral tegmental commissure runs from one side of the tegmentum to the other and is established by approximately 8 cells in the dorsolateral tegmentum.

\section{Identification of neurons with posterior commissure axons}

Discrete application of diI to the posterior commissure in 27$28 \mathrm{hr}$ embryos $(n=21)$ revealed that the posterior commissure consists of axons from 2 nuclei in addition to the dorsolaterally located nuc PC: a lateral cluster of up to 12 anterior tegmental cells found medial to the eye and dorsal to the TPOC, and a ventral cluster of up to 12 anterior tegmental neurons found just anterior to the nuc MLF (Fig. 9). Examination of the backlabeled cells from a diI application lateral to the dorsal midline $(n=5)$ showed that the ventral tegmental clusters of neurons have axons which course dorsally along the posterior commissure and cross the dorsal midline to the contralateral side (Fig. 10). Intracellular injections of LY into individual ventral tegmental cells $(n=2)$ confirmed that they project a single axon which travels dorsally into the posterior commissure and terminates in a lateral position on the other side (Fig. 10). In the 5 cases where tegmental cells contralateral to the diI injection site were examined, only the ventral cluster was labeled. This may be due to capriciousness of the labeling method or separation of axons from the lateral and ventral clusters near the application site, or it may indicate that the lateral cluster neurons do not cross the dorsal midline. DiI injections into the posterior commissure also labeled axons which extended anteriorly from the anterior tegmentum (Figs. 9, 10). At present the origin of these axons is unknown.

\section{Pathfinding by nuc PC growth cones}

Intracellular LY injection of individual cells $(n=31)$ in the nuc PC at various stages (20-28 hr) of development with LY demonstrated that nuc PC cells project a single growth cone which follows a stereotyped pathway (Fig. 11). At $20 \mathrm{hr}$, nuc PC neurons have projected growth cones approximately $20 \mu \mathrm{m}$ ventral to the nuc PC. By 23-24 hr, the ventrally directed growth cones have reached the anterior tegmentum. At this time, these growth cones have a complex morphology with numerous filopodia. DIC observation of the site of these growth cones showed that they are within the anterior tegmental clusters of neurons (see below) where the TPOC bundles, MLF, and the ventral tegmental commissure meet (data not shown). Despite the presence of several pathways at this site, the growth cones turn posteriorly and extend to the posterior region of the tegmentum and eventually into the hindbrain. DiI back-labeling of nuc PC neurons demonstrated that their axons are found in close association 

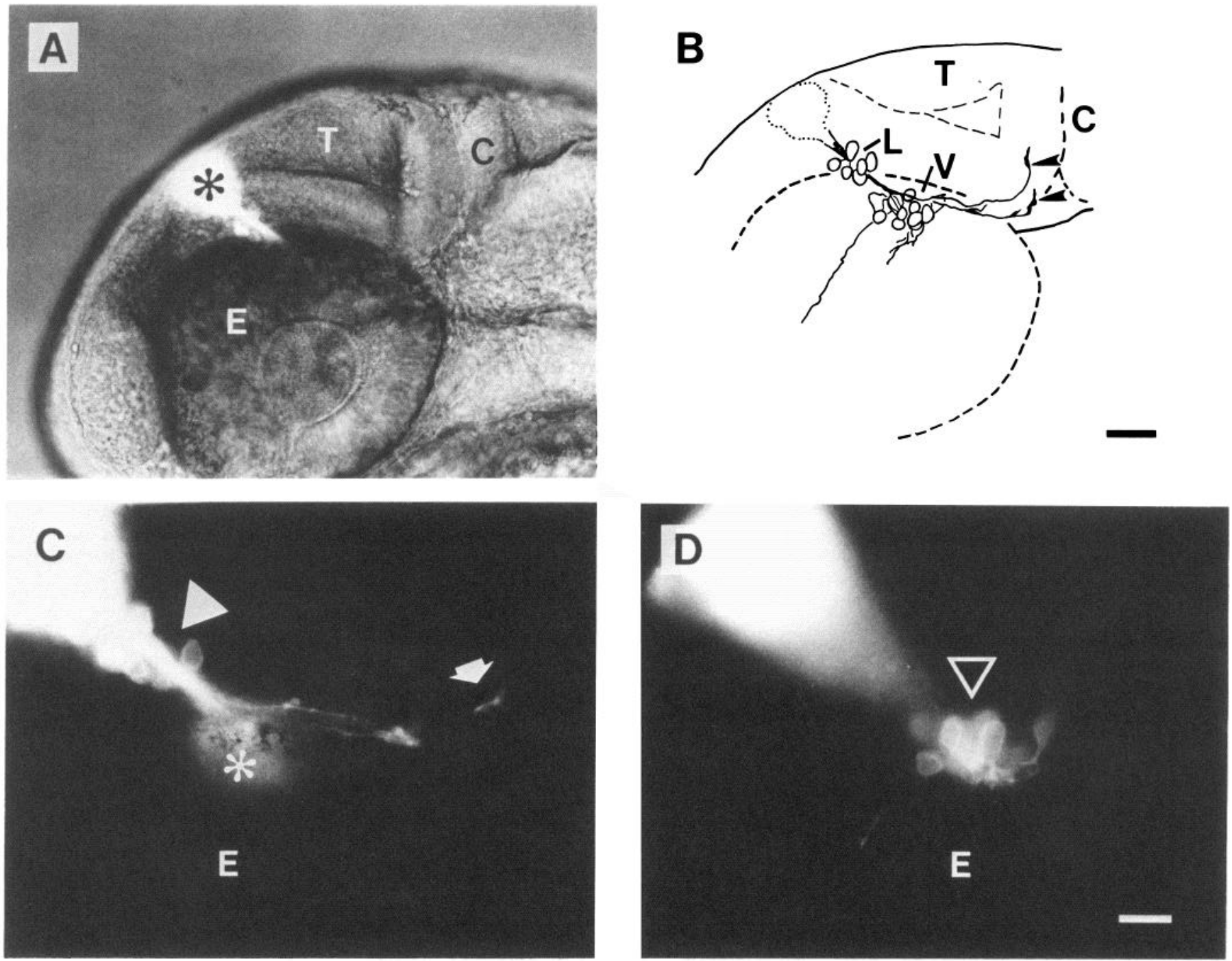

Figure 9. Identification of the lateral and ventral tegmental cluster of neurons which project axons into the posterior commissure in a $28 \mathrm{hr}$ embryo by diI labeling. $A$, Combined transillumination and epifluorescence photograph. Asterisk, site of diI injection which appears much larger than it is due to the haloing of the site, which is out of focus. $B$, Drawing of diI-labeled neurons from $A$. $L$, lateral tegmental cluster; $V$, ventral tegmental cluster; arrowheads, growth cones of neurons with axons in the posterior commissure; dotted region, diI injection site. Scale bar (in $B$ ) for $A$ and $B, 40 \mu \mathrm{m}$. $C$, Fluorescence micrograph of the lateral tegmental cluster (closed triangle). Asterisk, ventral tegmental cluster out of focus; arrow, growth cone. $D$, Fluorescence micrograph of the ventral tegmental cluster (open triangle). Scale bar (in $D$ ) for $C$ and $D, 20 \mu \mathrm{m}$.

Figure 10. Posterior commissure neurons of the ventral tegmental cluster have a single axon in the posterior commissure. Drawings of dye-filled lateral and ventral cluster tegmental cells with posterior commissural axons. $A, \mathrm{LY}$ filled ventral cluster neuron from a 28 hr embryo. Contralateral eye is outlined in dashes; axon on the contralateral side is dashed. $B$, DiI-labeled neurons in a $28 \mathrm{hr}$ embryo. Site of injection is denoted by dashed oval. Neurons of the ventral tegmental cluster contralateral to injection site are filled in; somata of the lateral and ventral clusters ipsilateral to injection site are outlined; ipsilateral axons are dashed. Scale bar, 50 $\mu \mathrm{m}$.
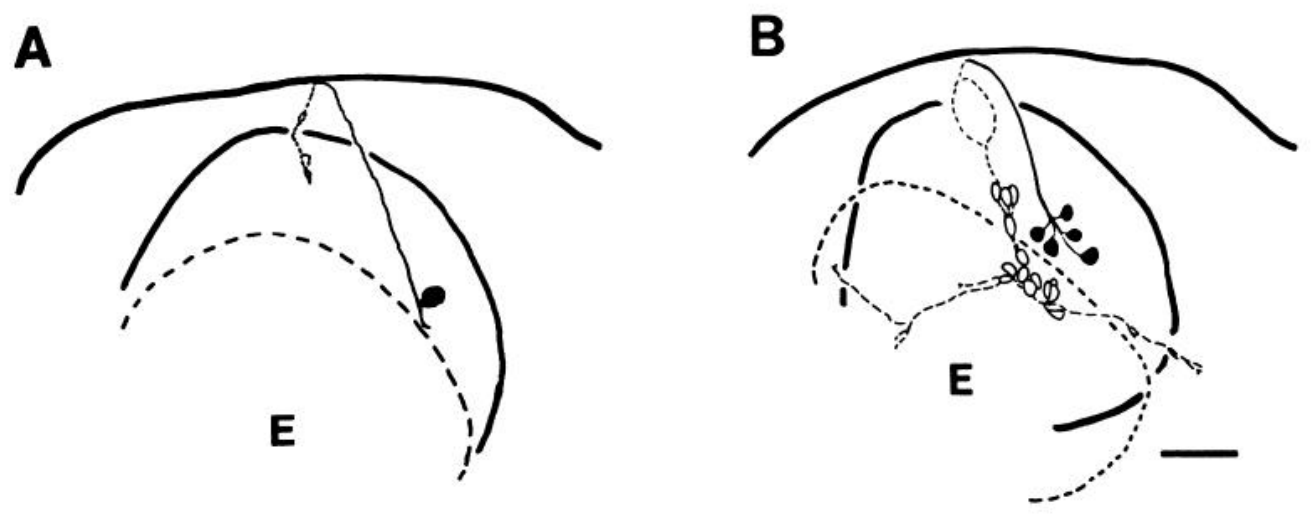

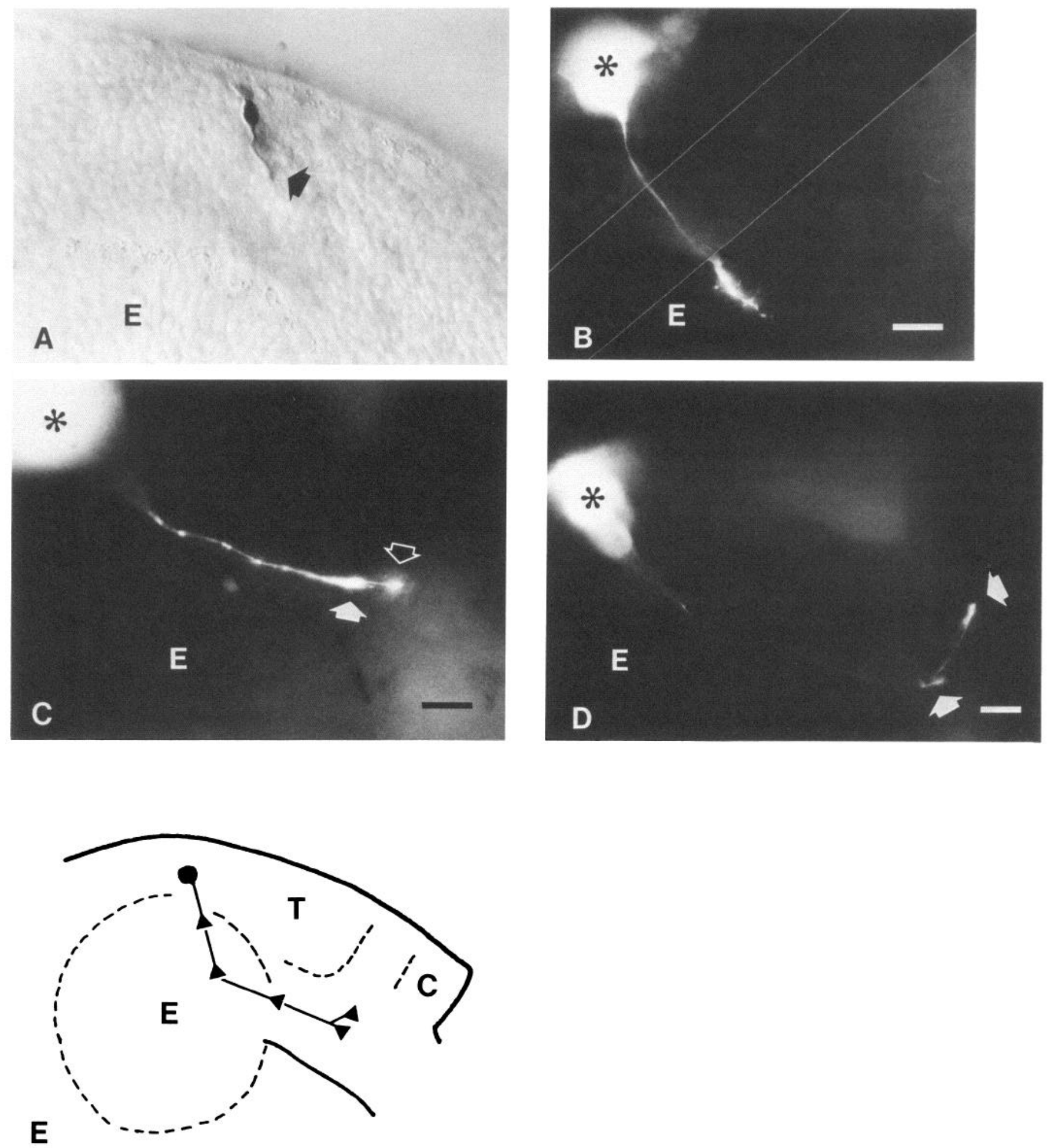

Figure 11. Nuc PC neurons project a single growth cone which follows a stereotyped, cell specific pathway. A, Micrograph of a photooxidized, LY-filled nuc PC neuron with a single, ventrally directed growth cone from a $20 \mathrm{hr}$ embryo. Arrow, growth cone. $B$, Fluorescence micrograph of a LY-filled nuc PC neuron with its growth cone in the anterior tegmentum where the posterior commissure, TPOC, ventral tegmental commissure, and MLF intersect from a 23-24 hr embryo. Asterisk, out-of-focus cell body of nuc PC neuron. Scale bar (in $B$ ) for $A$ and $B, 20 \mu \mathrm{m}$. $C$, Fluorescence micrograph of 2 nuc PC growth cones (filled and open arrows) from 2 nuc PC cells extending in close association with each other in a 26 hr embryo. Asterisk, location of the 2 nuc PC cell bodies. $D$, Fluorescence micrograph from a $28 \mathrm{hr}$ embryo showing a nuc PC cell with a growth cone extending dorsally at the posterior boundary of the midbrain and a growth cone extending posteriorly. Arrows, growth cones of filled cell. Scale bar (in $D$ ) for $C$ and $D, 20 \mu \mathrm{m}$. E. Schematic diagram showing the approximate locations of the growth cones shown in $A-D$. 

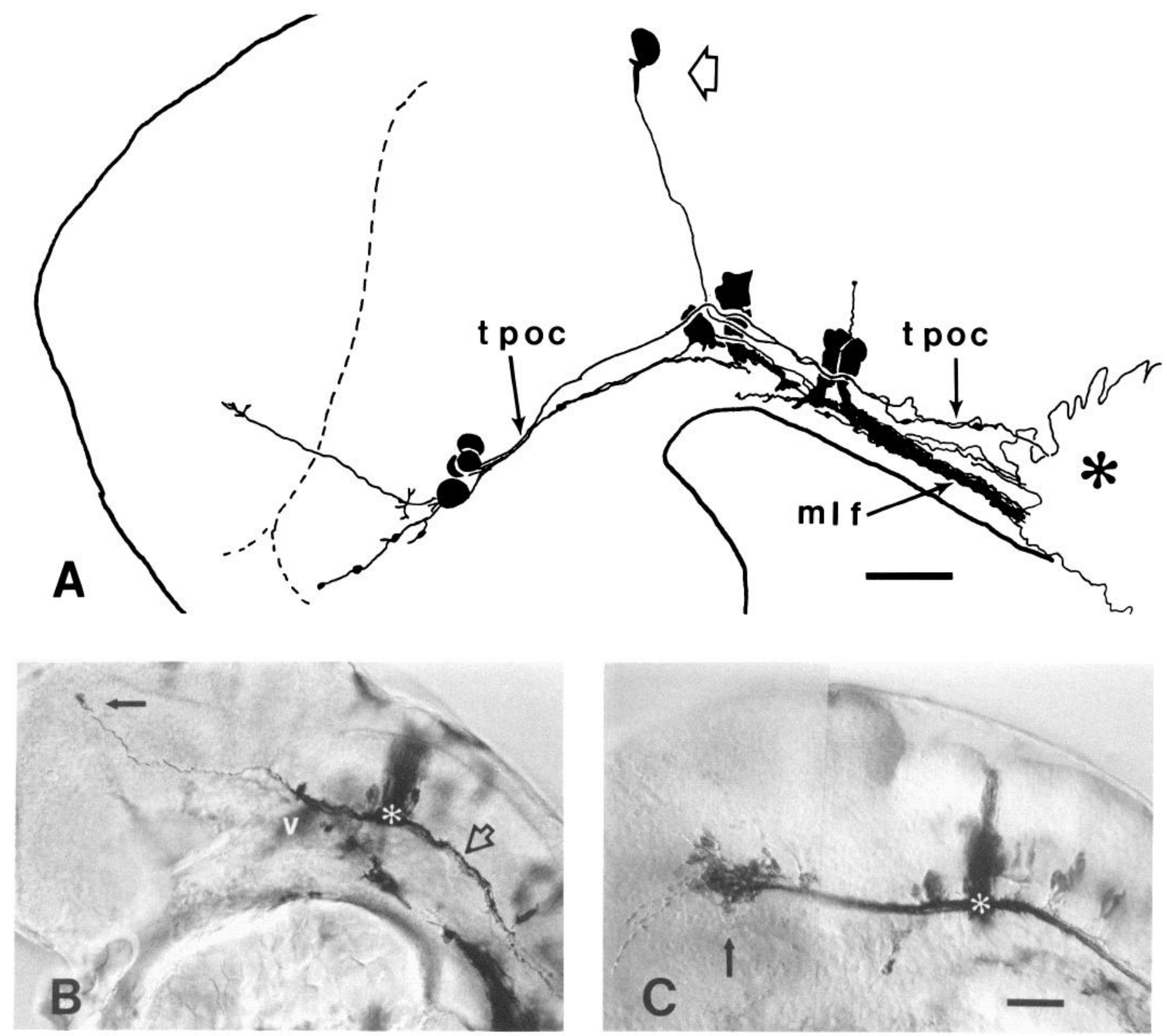

Figure 12. Longitudinal axons of nuc PC neurons are located in a dorsal bundle of the TPOC in the dorsolateral tegmentum. A, Camera lucida drawing of the axons in the MLF and TPOC back-labeled by an application of dil into the posterior tegmentum/anterior hindbrain (asterisk). The nuc PC (open arrow) longitudinal axon can be seen to intermingle with an axon in a dorsal bundle of the TPOC. Scale bar, $25 \mu \mathrm{m}$. In all panels, dil labeling was followed by photooxidation in $28 \mathrm{hr}$ embryos. B, Micrograph of a nuc PC neuron (closed arrow) back-filled by application of dil to the dorsolateral hindbrain (asterisk). This application also labeled the DLF (open arrow) and the trigeminal ganglion cells ( $V$ ), which are out of the focal plane and project into the DLF. The dark cells in the lower right are melanophores. $C$, Micrograph of the nuc MLF (arrow) labeled by a ventromedial application of diI into the hindbrain (asterisk). Scale bar (in $C$ ) for $B$ and $C, 50 \mu \mathrm{m}$.

with each other ( $n=3$; data not shown). In 4 of 5 cases, intracellular fills of pairs of nuc PC neurons showed that this likely occurs because the growth cones of nuc PC neurons extend in close association with the axons of other nuc PC neurons (Fig. $11 C$ ).

Labeling axons with the acetylated tubulin antibody indicated that the longitudinal axons of the nuc PC cells follow preexisting longitudinal tracts (Fig. 8). The identity of the longitudinal tracts (dorsal bundles of the TPOC or the MLF) was determined by diI labeling of the TPOC and the MLF in $28 \mathrm{hr}$ embryos $(n=$ 4). In these embryos, the nuc PC axons were found extending longitudinally in a dorsal bundle of the TPOC (Fig. 12A). In concordance with this, diI application to the dorsolateral hindbrain, the location of the dorsal bundles of the TPOC, labeled TPOC neurons in the diencephalon and nuc PC neurons $(n=$ $13)$. Figure $12 B$ shows a case in which a single nuc $P C$ neuron and the DLF and trigeminal ganglion neurons which project into the DLF (Kimmel et al., 1985) were labeled. This was expected since the dorsal bundles of the TPOC merge into the DLF in the hindbrain. Finally, ventromedial applications of diI into the hindbrain labeled the nuc MLF but not the nuc PC neurons ( $n=11$; Fig. $12 C$ ). 
A

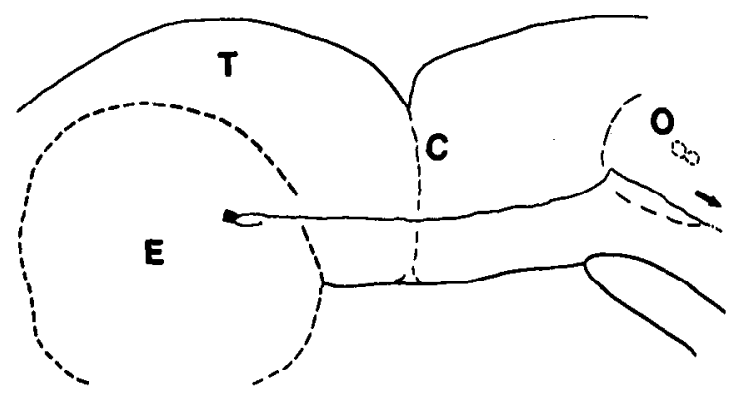

B

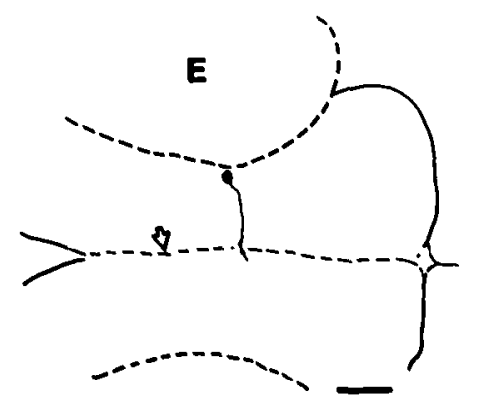

Figure 13. Nuc MLF and ventral tegmental commissural neurons have axons with stereotyped trajectories. Drawings of LY-filled brain neurons. $A$, Side view of a nuc MLF neuron in a 28 hr embryo. Arrowindicates that the axon extends beyond the drawing. $O$, otocyst. $B$, Ventral view of an anterior tegmental neuron with growth cone crossing the ventral midline in the tegmental commissure in a $26 \mathrm{hr}$ embryo. Open arrow, ventral midline; anterior is left. Scale bar (in $B$ ) for $A$ and $B, 50 \mu \mathrm{m}$.

\section{Anterior tegmental neurons}

The anterior tegmentum contains 4 groups of neurons at approximately 26-28 hr of development. Each group of neurons appears to have axons with cell-specific trajectories. The lateral and ventral tegmental clusters of neurons have axons in the posterior commissure as described above. Another set of neurons constitute the nuc MLF which projects axons posteriorly into the MLF (see above). Intracellular injections of these neurons ( $n=$ 4) with LY further showed that at least some nuc MLF neurons have only a single axon at $28 \mathrm{hr}$ (Fig. 13A). Intracellular LY fills of a fourth group of anterior tegmental neurons $(n=5)$ demonstrated that they only have axons which extend toward the ventral midline and cross to the other side of the tegmentum in the ventral tegmental commissure (Fig. 13B). It is not clear whether the distribution of these 3 groups of neurons overlaps. However, in acetylated tubulin-labeled embryos where it was possible to trace axons back to their cell bodies, the tegmental neurons with posterior commissure axons were among the most anterior cells in the tegmentum. Those with ventral tegmental commissure axons were the most dorsolateral and the nuc MLF neurons were the most medial and posterior.

\section{Discussion}

\section{Organization of the axonal pathways in the brain of vertebrate embryos}

The embryonic brain of zebrafish consists of a simple and stereotyped set of axonal tracts which are initially established by a relatively small number of neurons. We identified the early brain tracts by application of the acetylated tubulin antibody and by making large applications of diI to various regions of the embryonic brain. Essentially the same pattern of tracts was seen in the embryonic zebrafish brain by back-filing neurons with HRP (Wilson et al., 1990).

The conclusion that the acetylated tubulin antibody probably labels all the early tracts in the zebrafish embryo is based on several observations. First, all the axonal tracts seen in EM sections of the embryonic brain corresponded to the tracts labeled by the antibody in semi-thin sections similar to the EM sections. The EM sections also demonstrated that these early axonal profiles contained microtubules and $\alpha$-tubulin has been shown to be acetylated in the polymeric form and deacetylated upon depolymerization (Black et al., 1989). Second, large applications of dil into various regions of the brain labeled the same pattern of tracts labeled by the antibody. Third, the same pattern of early tracts has been revealed by back-filling neurons with HRP in the zebrafish embryo (Wilson et al., 1990). Fourth, the acetylated tubulin antibody labeled all the neuronal cell types which had been identified by axonal tracer dyes and intracellular dye injections in the spinal cords of embryonic zebrafish (Bernhardt et al., unpublished observations; Kuwada et al., 1990). Fifth, previous investigations using silver staining techniques to label axons in the embryonic amphibian revealed a similar set of early brain tracts (see below). Sixth, in the nematode the antibody heavily labels a subset of neurons but apparently lightly labels all other axons (Siddiqui et al., 1989).

Our results generally confirm and extend the observations of Coghill $(1926,1930)$ and Herrick $(1937,1938)$ who studied the pattern of axonogenesis in embryonic amphibian brains. These investigators found that the carlicst axonal tracts were found in the ventral diencephalon, anterior tegmentum, and specific regions of the telencephalon. This corresponds to the earliest tracts in the zebrafish embryonic brain.

The early tracts in the CNS are organized into a simple set of longitudinal tracts connected by commissures in many different animals, including nematodes, insects, amphibians, and fish (Herrick, 1937, 1938; Albertson and Thomson, 1976; White et al., 1976; Thomas et al., 1984). The highly complex pattern of tracts found in the mature CNS emerge from the initial, simple pattern of tracts as more neurons differentiate and additional tracts are formed. The commonality of the initial framework of tracts may indicate that different animals share similar strategies for the formation of tracts. For example, in the insect CNS the early pattern of discrete commissural and longitudinal tracts is transformed into one in which all tracts are contiguous and collected into 2 commissures and a bilateral pair of longitudinal connectives (Bastiani et al., 1984). This occurs both by the establishment of new tracts among the already established tracts and the addition of axons to preexisting tracts. The position of each tract is not correlated with time of formation of the tract. Likewise, in the spinal cord of zebrafish embryos there is an initial set of discrete fascicles (Kuwada et al., 1990). The contiguous marginal zone forms as a result of addition of new axons to the early fascicles and by the extension of longitudinal axons in between the early fascicles. In neither case is the mature pattern of tracts established predominantly by new tracts following earlier tracts nor by a spatiotemporal mechanism. A similar pattern of tract formation may also occur in the zebrafish brain. In the tegmentum, several discrete bundles of longitudinal axons are evident by $28 \mathrm{hr}$ of development. However, in adult teleosts the ventral margin of the tegmentum is a continous zone 
of axons (Ariens Kappers et al., 1936; Nieuwenhuys and Pouwels, 1983).

\section{Pathfinding in the embryonic vertebrate brain}

Acetylated tubulin labeling of the earliest stages of axonogenesis in the zebrafish brain indicated that many neurons project axons in a defined direction appropriate for cell clusters. Although we cannot rule out the possibility that in some cases aberrant axons with unacetylated microtubules may have been missed, this is unlikely for at least one cluster of neurons, the nuc PC cells. Intracellular LY fills of nuc PC cells at various stages of axonogenesis demonstrated that they project a single growth cone which navigates through the brain by following a stereotyped and precise pathway. Furthermore, nuc PC neurons do not extend exuberant axons by bifurcating at sites where several tracts intersect. This is similar to pathfinding by spinal neurons in fish embryos (Eisen et al., 1986; Kuwada, 1986; Kuwada et al., 1990, unpublished observations). No early brain or spinal neuron in the fish embryos so far analyzed, projects exuberant axons which are eliminated at a later stage during embryogenesis, as do numerous neurons in the mammalian CNS (Cowan et al., 1984). However, only the earliest neurons in the zebrafish brain and spinal cord have been studied and, in most cases, only through embryogenesis. Therefore, it is possible that the early differentiating neurons may project exuberant axons following embryogenesis and/or that later-developing neurons may project exuberant axons.

Intracellular LY injections and diI backfills demonstrate that many of the early brain neurons of zebrafish embryos have stereotyped axonal trajectories and suggest that they are established by growth cones following specific pathways. This was confirmed for the growth cones of the nuc PC neurons. The stcreotyped pathways of growth cones from neurons in the vertebrate brain and spinal cord (Eisen et al., 1986; Kuwada, 1986; Landmesser, 1988; Kuwada et al., unpublished observations) may indicate that, like growth cones in the insect CNS (Goodman et al., 1984), some vertebrate growth cones selectively choose their pathways at sites where several pathways intersect. Nuc PC growth cones extend ventrally to the region of the anterior tegmentum where the posterior commissure, TPOC, MLF, and ventral tegmental commissure meet. Since there are axons which traverse this intersection from a number of different directions by this time, the nuc PC growth cones could, in principle, turn anteriorly or posteriorly into the TPOC, posteriorly into the MLF, or toward the ventral midline in the ventral tegmental commissure. Yet the growth cones from the nuc PC always turn posteriorly into the TPOC. Additionally, in the anterior tegmentum there are 4 groups of neurons which have axons in only 1 of the 4 pathways which converge at this intersection: the lateral and ventral clusters of posterior commissure neurons have axons which run dorsally in the posterior commissure, the ventral commissural neurons have axons which cross the midline in the ventral commissure, and the neurons in the nuc MLF have caudally running axons in the MLF. This suggests the hypothesis that the growth cones of these neurons choose their pathways at this intersection in the embryonic zebrafish brain. Verification of pathway selection by identified growth cones awaits electron microscopy of these growth cones at this intersection to see if growth cones have access to the inappropriate as well as appropriate pathways. Analysis of mechanisms of pathway selection await manipulation of the embryonic pathways these growth cones normally encounter.

\section{References}

Albertson DG, Thomson JN (1976) The pharynx of Caenorhabditis elegans. Phil Trans R Soc London [Biol] 275:299-325.

Ariens Kappers CU, Huber GC, Crosby EC (1936) The comparative anatomy of the nervous system of vertebrates including man, $\mathrm{Vol} 2$, pp 903-939. New York: Macmillan.

Bastiani M, Pearson KG, Goodman CS (1984) From embryonic fascicles to adult tracts : organization of neuropile from a developmental perspective. J Exp Biol 112:45-64.

Black MM, Baas PW, Humphries S (1989) Dynamics of $\alpha$-tubulin deacctylation in intact ncurons. J Neurosci 9:358-368.

Blair SS, Murray MA, Palka J (1987) The guidance of axons from transplanted neurons through aneural Drosophila wings. J Neurosci 7:4165-4175.

Caudy M, Bentley D (1986a) Pioneer growth cone morphologies reveal proximal increases in substrate affinity within leg segments of grasshopper embryos. J Neurosci 6:364-379.

Caudy M, Bentley D (1986b) Pioneer growth cone steering along a series of neuronal and non-neuronal cues of different affinities. J Neurosci 6:1781-1795.

Coghill, GE (1926) Correlated anatomical and physiological studies of the growth of the nervous system of Amphibia. VI. The mechanism of integration in Amblystoma punctatum. J Comp Neurol 40:95-1 52.

Coghill GE (1930) Correlated anatomical and physiological studies of the growth of the nervous system of Amphibia. IX. The mechanism of association in Amblystoma punctatum. J Comp Neurol 51:147168.

Cowan WM, Fawcett JW, O'Leary DDM, Stanfield BB (1984) Regressive events in neurogenesis. Science 225:1258-1265.

Dodd J, Jessell TM (1988) Axon guidance and the patterning of neuronal projections in vertebrates. Science 242:692-699.

Easter SS, Taylor JSH (1989) The development of Xenopus retinotectal pathway: optic fibers join a pre-existing tract. Development 107:553-573.

Eisen JS, Myers PZ, Westerfield M (1986) Pathway selection by growth cones of identified motoneurons in live zebrafish embryo. Nature 320 : 269-271.

Eisen JS, Pike SH, Debu B (1989) The growth cones of identified motoneurons in embryonic zebrafish select appropriate pathways in the absence of specific cellular interactions. Neuron 2:1097-1104.

Goodman CS, Bastiani MJ, Doe CQ, du Lac S, Helfand SL, Kuwada JY, Thomas JB (1984) Cell recognition during neuronal development. Science 225:1271-1279.

Herrick CJ (1937) Development of the brain of Amblystoma in early functional stages. J Comp Neurol 67:381-422.

Herrick CJ (1938) Development of the cerebrum of Amblystoma during early swimming stages. J Comp Neurol 68:203-241.

Honig MG, Hume RI (1986) Fluorescent carbocyanine dyes allow living neurons of identified origin to be studied in long-term cultures. J Cell Biol 103:171-187.

Jan LY, Jan YN (1982) Antibodies to horseradish peroxidase as specific neuronal markers in Drosophila and in grasshopper embryos. Proc Natl Acad Sci USA 79:2700-2704.

Johnston JB (1911) The telencephalon of ganoids and teleosts. J Comp Neurol 21:489-591.

Kimmel CB, Powell SL, Metcalfe WK (1982) Brain ncurons which project to the spinal cord in the young larvae of zebrafish. J Comp Neurol 205:112-127.

Kimmel CB, Metcalfe WK, Schabtach E (1985) T reticular interneurons: a class of serially repeating cells in the zebrafish hindbrain. $J$ Comp Neurol 233:365-376.

Kuhlenbeck H (1977) The central nervous system of vertebrates: Vol 5, part 1: derivatives of the prosencephalon: diencephalon and telencephalon, pp 170-180. New York: Karger.

Kuwada JY (1986) Cell recognition by neuronal growth cones in a simple vertebrate embryo. Science 233:740-746.

Kuwada JY, Bernhardt RR, Chitnis AB (1990a) Pathfinding by identified growth cones in the spinal cord of zebrafish embryos. J Neurosci 10:1299-1308.

Landmesser L (1988) Peripheral guidance cues and formation of specific motor projections in the chick. In: From message to mind (Easter S, Barald K, Carlson B, eds), pp 121-133. Sunderland, MA: Sinauer.

Maranto AR (1982) Neuronal mapping: a photooxidation reaction Lucifer Yellow useful for election microscopy. Science 217:953-955. 
Myers PZ, Eisen JS, Westerfield M (1986) Development and axonal outgrowth of identified motoneurons in the zebrafish. J Neurosci 6 : 2278-2289.

Nieuwenhuys R, Pouwels E (1983) The brain stem of actinopterygian fishes. In: Fish neurobiology, Vol 1 (Northcutt RG, Davis RE, eds), pp 25-87. Ann Arbor, MI: University of Michigan Press.

Palka J (1986) Neurogenesis and axonal pathfinding in invertebrates. Trends Neurosci 9:482-485.

Patterson PH (1988) On the importance of being inhibited, or saying no to growth cones. Neuron 1:263-267.

Patel NH, Martin-Blanco E, Coleman KG, Poole SJ, Ellis MC, Kornberg TB, Goodman CS (1989) Expression of engrailed protein in arthropods, annelids, and chordates. Cell 58:955-968.

Piperno G, Fuller MT (1985) Monoclonal antibodies specific for an acetylated form of $\alpha$-tubulin recognize the antigen in cilia and flagella from a variety of organisms. J Cell Biol 101:2085-2094.

Roberts A, Dale N, Ottersen OP, Storm-Mathisen J (1987) Early development of neurons with GABA immunoreactivity in the CNS of Xenopus laevis embryos. J Comp Neurol 261:435-449.

Siddiqui SS, Aamondt E, Rastinejad F, Culotti J (1989) Anti-tubulin monoclonal antibodies that bind to specific neurons in Caenorhabditis elegans. J Neurosci 9:2963-2972.

Stanfield BB, O'Leary DDM (1985) The transient corticospinal projection from the occipital cortex during the postnatal development of the rat. J Comp Neurol 238:236-248.

Stanfield BB, O’Leary DDM, Fricks C (1982) Selective collateral elimination in early postnatal development restricts cortical distribution of rat pyramidal tract neurons. Nature 298:371-373.

Taghert PH, Bastiani MJ, Ho RK, Goodman CS (1982) Guidance of pioncer growth concs: filopodial contacts and coupling revealed with an antibody to Lucifer Yellow. Dev Biol 94:391-399.

Thomas JB, Bastiani MJ, Bate M, Goodman CS (1984) From grasshopper to Drosophila: a common plan for neuronal development. Nature 310:203-207.

White JG, Southgate E, Thomson JN, Brenner S (1976) The structure of the ventral nerve cord of Caenorhabditis elegans. Phil Trans R Soc London [Biol] 275:327-348.

Wilson SW, Ross L, Parrot T, Easter SS (1990) The development of a simple scaffold of axon tracts in the brain of embryonic zebrafish, Brachydanio kerio. Development 108:121-145. 